\title{
Deficiency of DNA fragmentation factor 45 results in reduced oocyte apoptosis in response to doxorubicin
}

\author{
Mei Dong ${ }^{1, *}$, Yunxia Fan ${ }^{1, *}$, Nicholas J Toepfer ${ }^{1}$, Jianhua Zhang ${ }^{1,2}$ \\ ${ }^{1}$ Department of Cell Biology, Neurobiology and Anatomy, University of Cincinnati College of medicine, Cincinnati, OH 45267, \\ $U S A$
}

Cell Research (2007) 17:735-736. doi: 10.1038/cr.2007.62; published online 31 July 2007

\section{Dear Editor:}

Apoptosis plays a prominent role in ovarian development and function [1-4]. During follicle development, the vast majority of follicles undergo atresia as a consequence of apoptosis of constituent oocyte or follicular cells, or both, failing to complete the maturation process. Atresia occurs at all stages of follicular development during the growth and development of follicles. Atresia of the primordial and primary follicles is initiated by oocyte apoptosis, followed by the death of the granulosa cells [1-4]. In comparison, the reduction of the large number of growing follicles to a single ovulatory follicle is achieved primarily by the cell death of granulosa cells [1-4]. Members of the Bcl-2 and caspase families play important roles in the regulation of ovarian cell death, both in follicle development and in response to chemotherapeutic agents [5].

DNA fragmentation factor (DFF), consisting of DFF40 (also called caspase-activated Dnase (CAD)) and DFF45 (also called inhibitor of caspase-activated Dnase (ICAD)), cleaves DNA into nucleosomal-sized fragments during apoptosis [6]. To understand the in vivo function of DFF45, we previously generated DFF45 mutant mice [7, $8]$. We found that DFF45-deficient cells are more resistant to apoptosis than wild-type control cells after exposure to several apoptotic stimuli [7-9]. DFF45 mutant mice exhibit enhanced learning and memory $[9,10]$. Collectively, these data indicated a critical role of DFF45 in normal apoptosis and tissue homeostasis.

To examine whether DFF45 gene disruption affects

\footnotetext{
*These two authors contributed equally to this work.

Correspondence: Jianhua Zhang

${ }^{2}$ Present address: Department of Pathology, 961 Sparks Center, 1530 3rd Ave S, University of Alabama at Birmingham, Birmingham, AL 352940017, USA

Tel: +1-205-996-5153; Fax: +1-205-934-6700

E-mail: zhanja@uab.edu
}

oocyte apoptosis, we collected oocytes from wild-type or DFF45-/- females. The oocytes were then denuded and cultured with or without $200 \mathrm{nM}$ of the anti-cancer drug, doxorubicin (DXR), to induce apoptosis.

The superovulation rate of $D F F 45$-/- females was comparable with that of wild-type females $(18.00 \pm 2.69$ vs. $16.63 \pm 3.42$ oocytes/mouse, respectively; mean $\pm \mathrm{SEM}$; $\mathrm{n}=8$ mice/genotype). Although no significant difference $(p>0.05)$ was found in spontaneous apoptosis in oocytes retrieved from wild-type vs DFF 45-/- female mice (Figure $1 \mathrm{~A}$ and $1 \mathrm{~B}$ ), the incidence of DXR-induced apoptosis was significantly lower in oocytes harvested from DFF45-/female mice than that from wild-type sisters, when treated in parallel with DXR for $20 \mathrm{~h}$. By $48 \mathrm{~h}$ in culture, apoptosis, either spontaneous or drug-induced, was similar in oocytes harvested from wild-type and DFF45-/- females (Figure 1B).

We analyzed serial ovarian sections from wild-type and DFF45-/- female mice to examine the potential effect of the loss of DFF45 on follicle development. At 18 months of age, we found that DFF45-/- females possess more numbers of both non-atretic secondary follicle and graafian follicle than wild-type females (Supplementary Figure 1). Ovaries of wild-type mice contained follicles with many TUNEL-positive cells (Supplementary Figure 1B and 1C). The number of TUNEL-positive cells per follicle was significantly lower $(p<0.05)$ in DFF45-/- mice $(25.50 \pm$ $5.00)$, compared with follicles in wild-type mice (53.80 \pm 10.63 ).

In 4-day-old females, we found no significant difference in the number of primordial and primary follicles between wild-type and DFF45-/- females (Supplementary Figure $2 \mathrm{~A}$ ), suggesting that follicle endowment in wild-type and DFF45-/- female mice shortly after birth were comparable. At 14 days of age, the number of late primary follicles was slightly increased in DFF45-/- females compared with that in wild-type females (Supplementary Figure 2B). At 
A

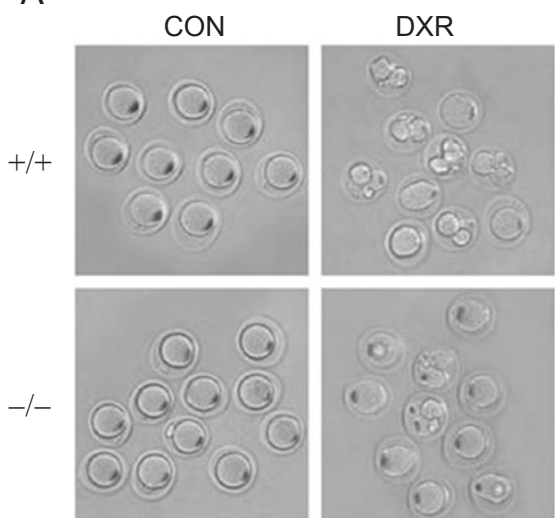

B

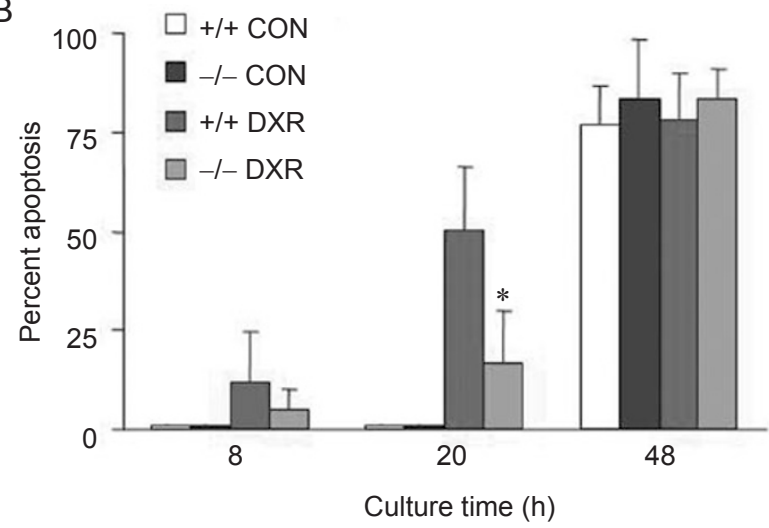

Figure 1 DFF45-/- oocytes exhibit decreased DXR-induced cell death compared with wild-type oocytes. Oocytes collected from wild-type (+/+) and DFF45-deficient (-/-) female mice were cultured in vitro in the absence or presence of $200 \mathrm{nM}$ DXR for 8, 20, and $48 \mathrm{~h}$. At the termination of culture, the occurrence of apoptosis was assessed first by observation of nuclear fragmentation and then by DAPI staining. (A) Representative images capturing the cellular morphology in pools of wild-type and DFF45-deficient oocytes cultured without DXR for $20 \mathrm{~h}(\mathrm{CON})$; and pools of wild-type and DFF45-deficient oocytes cultured with $200 \mathrm{nM}$ DXR for $20 \mathrm{~h}$ (DXR). (B) Bar graph of occurrence of apoptosis in oocytes from wild-type and DFF45-deficient mice after in vitro culture in the absence (CON) or in the presence of DXR (DXR) for 8, 20, and $48 \mathrm{~h}$. The experiments were repeated with $\mathrm{n}=3$ families for each genotype. Data are given as mean $+\mathrm{SEM}$. ${ }^{*} p<0.05$. Student's $t$-test is used for comparison between genotypes for the same treatment.

42 days of age, $D F F 45$-/- female mice possessed fewer early primary follicles (Supplementary Figure 2C) compared with those in wild-type counterparts. Overall, DFF45-/mice do not exhibit overt changes in follicle numbers at the age groups we examined, despite their having reduced number of TUNEL-positive cells (Supplementary Figure $2 \mathrm{D}-2 \mathrm{~F})$. One likely possibility is that other nucleases are more important for developmental cell death in the ovary. Another possibility is that nucleases are not required for developmental cell death in the ovary.

The ovarian volumes in the wild-type and DFF45-/- female mice $(n=10$, each) at 4 days, 14 days, 42 days, and 18 months of age are not significantly different between genotypes $(p>0.05)$ (data not shown). In conclusion, this study demonstrated the functional importance of DFF45 in execution of apoptosis in cultured oocytes in response to chemotherapeutic agents, but DFF45 is not critically required for ovarian follicular cell maturation and development.

\section{Acknowledgments}

We thank our colleagues Dr Ming Xu for reagents and discussion, Dr Nancy Kleene for microscopy assistance, and Zhou Fang for technical support. This work was supported by DOD (Department of Defense Ovarian Cancer Research Program, JZ).

\section{References}

1 Kaipia A, Hsueh AJ. Regulation of ovarian follicle atresia. Annu
Rev Physiol 1997; 59:349-363.

2 Hsueh AJ, Billig H, Tsafriri A. Ovarian follicle atresia: a hormonally controlled apoptotic process. Endocr Rev 1994; 15:707724.

3 Tilly JL. Commuting the death sentence: how oocytes strive to survive. Nat Rev Mol Cell Biol 2001; 2:838-848.

4 Morita Y, Tilly JL. Oocyte apoptosis: like sand through an hourglass. Dev Biol 1999; 213:1-17.

5 Kim MR, Tilly JL. Current concepts in Bcl-2 family member regulation of female germ cell development and survival. Biochim Biophys Acta 2004; 1644:205-210.

6 Liu X, Zou H, Slaughter C, Wang X. DFF, a heterodimeric protein that functions downstream of caspase-3 to trigger DNA fragmentation during apoptosis. Cell 1997; 89:175-184.

7 Zhang J, Wang X, Bove KE, Xu M. DNA fragmentation factor 45-deficient cells are more resistant to apoptosis and exhibit different dying morphology than wild-type control cells. J Biol Chem 1999; 274:37450-37454.

8 Zhang J, Liu X, Scherer DC, van Kaer L, Wang X, Xu M. Resistance to DNA fragmentation and chromatin condensation in mice lacking the DNA fragmentation factor 45. Proc Natl Acad Sci USA 1998; 95:12480-12485.

9 Zhang J, Lee H, Agarwala A, Wen LD, Xu M. Dna fragmentation factor 45 mutant mice exhibit resistance to kainic acidinduced neuronal cell death. Biochem Biophys Res Commun 2001; 285:1143-1149.

10 Slane JM, Lee HS, Vorhees CV, Zhang J, Xu M. DNA fragmentation factor 45 deficient mice exhibit enhanced spatial learning and memory compared to wild-type control mice. Brain Res 2000; 867:70-79.

(Supplementary Information is linked to the online version of the paper on the Cell Research website.) 